\title{
New information about Albert Einstein's brain
}

\author{
Dean Falk* \\ Department of Anthropology, Florida State University, Tallahassee, FL, USA
}

Edited by:

Steven M. Platek, Georgia Gwinnett

College, USA

Reviewed by:

Keith Erickson, Georgia Gwinnett

College, Lawrenceville, USA

Andrew C. Gallup, Binghamton

University, USA

Katerina Semendeferi, University of

California-San Diego, USA

Harry Jerison, University of California,

USA

\section{*Correspondence:}

Dean Falk, Department of

Anthropology, Florida State University,

1847 W. Tennessee Street, Tallahassee,

FL 32306-7772, USA.

e-mail:dfalk@fsu.edu
In order to glean information about hominin (or other) brains that no longer exist, details of external neuroanatomy that are reproduced on endocranial casts (endocasts) from fossilized braincases may be described and interpreted. Despite being, of necessity, speculative, such studies can be very informative when conducted in light of the literature on comparative neuroanatomy, paleontology, and functional imaging studies. Albert Einstein's brain no longer exists in an intact state, but there are photographs of it in various views. Applying techniques developed from paleoanthropology, previously unrecognized details of external neuroanatomy are identified on these photographs. This information should be of interest to paleoneurologists, comparative neuroanatomists, historians of science, and cognitive neuroscientists. The new identifications of cortical features should also be archived for future scholars who will have access to additional information from improved functional imaging technology. Meanwhile, to the extent possible, Einstein's cerebral cortex is investigated in light of available data about variation in human sulcal patterns. Although much of his cortical surface was unremarkable, regions in and near Einstein's primary somatosensory and motor cortices were unusual. It is possible that these atypical aspects of Einstein's cerebral cortex were related to the difficulty with which he acquired language, his preference for thinking in sensory impressions including visual images rather than words, and his early training on the violin.

Keywords: Albert Einstein, Brodmann's area 40, cortical asymmetries, parietal operculum, sulcal patterns, sensory and motor cortices

\section{INTRODUCTION}

Although the hypothesis that gross neuroanatomical features may reflect the mental abilities of exceptionally talented individuals has held a long fascination (Amunts et al., 2004; Witelson et al., 1999b), efforts to address it are frequently viewed with hesitation for several reasons: First, such studies have historically been associated with phrenology, which was rightfully dismissed at the end of the 19th century as a pseudoscience (Gould, 1981). Second, the extent to which one of the traditional foci of these studies, brain size, is correlated with intelligence is difficult to assess (Roth and Dicke, 2005). Finally, although sulcal patterns have also been of interest, sulci usually do not correlate precisely with the borders of functionally defined cytoarchitectonic fields (Amunts et al., 1999; Zilles et al., 1997), some of which have been associated with exceptional competence, e.g., the extraordinary cytoarchitectonic features in Broca's area of Emil Krebs (1867-1930) who was fluent in more than 60 languages (Amunts et al., 2004). Despite these caveats, however, gross sulcal patterns have been associated with enlarged cortical representations that subserve functional specializations in mammals including carnivores (Welker and Campos, 1963) and primates (Falk, 1982), in a phenomenon called the 'principle of proper mass' (Jerison, 1973). Raccoons, for example, have greatly enlarged primary somatosensory forepaw representations in which the various palm pad and digit areas are demarcated from one another by sulci, and this remarkable cortical morphology has been attributed to the fact that these animals use their forepaws extensively to explore their environments (Welker and Campos, 1963).

It is also well known that dramatic changes may occur in sensory and motor cortices during a human's lifetime as revealed by medical imaging studies of Braille readers and upper limb amputees, which show that the cerebral cortex can exhibit longterm adaptations, including enlargement or relocation of specific representations such as those for hands (Amunts et al., 1997). Further, gross cortical features entailing sulcal depths or patterns have been identified in people with exceptional abilities such as highly trained musicians (Amunts et al., 1997; Bangert and Schlaug, 2006; Schlaug, 2001) and the world-renowned physicist who is the subject of this report, Albert Einstein (Witelson et al., 1999a,b).

After his death in 1955 at 76 years of age, Albert Einstein's brain was removed from his body by Thomas Harvey (a pathologist), fixed, measured, photographed, and sectioned into 240 blocks that were embedded in celloidin (Lepore, 2001). Twelve sets of microscopic slides were prepared from the embedded blocks and distributed to various neuropathologists by Harvey (Lepore, 2001). It took decades, however, before papers began to appear on the histology or gross morphology of Einstein's brain. Although the neuron:glial ratio was determined to be significantly smaller in Einstein's left than right Brodmann's area (BA) 39 using the Kluver-Barrera stain (Diamond et al., 1985), this report has been criticized on methodological grounds (Hines, 1998). Another study determined that Einstein's prefrontal cortex had a greater neuronal density than those of normal controls because it packed approximately the same number of neurons into a thinner cortex (Anderson and Harvey, 1996), but one does not know the extent to which this was due to age, especially in people with superior intelligence (Shaw et al., 2006). Because the blocks of Einstein's brain were embedded in celloidin, histological studies using Golgi or other more revealing techniques would have been 
difficult if not impossible (Diamond et al., 1985). Einstein's cerebral cortex was thin (Anderson and Harvey, 1996) and had widened sulci, which were normal for his age (Magnotta et al., 1999). His brain mass of $1230 \mathrm{~g}$ (Witelson et al., 1999b) was also unexceptional. Gross anatomical studies of Einstein's brain included identifications for a number of sulci that were indicated on photographs (Witelson et al., 1999a,b), and provided measurements that were obtained directly from the brain, as well as others that were measured from calibrated photographs (Witelson et al., 1999b). The table in which the measurements appeared, however, did not indicate which ones were from each source, or when they were collected.

After being removed and processed, what remained of Einstein's previously whole brain were histological slides, fragments of brain stored in a jar of formaldehyde, unspecified measurements that Harvey obtained directly from the brain, and calibrated photographs (Lepore, 2001). Nevertheless, sulci may still be identified and interpreted from the extant photographs of Einstein's whole brain, in much the same way that cortical morphology is observed and studied on endocasts from fossils by paleoneurologists. It is hoped that the newly identified gyral and sulcal features reported below for Einstein's cerebral cortex will be of interest to future scholars. Despite the fact that a large portion of Einstein's cerebral cortex was superficially unremarkable, regions in and near his primary somatosensory and motor cortices were highly unusual, and it is tentatively suggested that these may have contributed to the neuroanatomical substrates for some of his remarkable abilities.

\section{MATERIALS AND METHODS}

Previously unrecognized gyral asymmetries and sulci are identified on published photographs of dorsal and lateral views of Einstein's whole brain (Witelson et al., 1999b), using traditional landmarks (Brodmann, 1909; Connolly, 1950; Ono et al., 1990; Yousry et al., 1997) and the terminology of Connolly (1950). To the extent possible, certain observations are quantified by comparing them to the range of measurements for normal humans published in Ono et al. (1990) and by referring to data published by Steinmetz et al. (1990), Witelson et al. (1999a), and Falk et al. (1991). Conflicting reports regarding Einstein's handedness have been resolved through photographic evidence that reveals Einstein held pens, manipulated objects, and played the violin like a right-hander (Wolff and Goodman, 2007). Results are tentatively interpreted in light of contemporary medical imaging studies (Bangert and Schlaug, 2006; Caulo et al., 2007; Falk et al., 1991; Steinmetz et al., 1990; Yousry et al., 1997) and published details about Einstein's linguistic and musical abilities (Bucky, 1992; Einstein, 1970; Hadamard, 1945; Wertheimer, 1959; Wolff and Goodman, 2007). The controversial question of whether or not Einstein had opercular cortices (Galaburda, 1999; Witelson et al., 1999a,b) is revisited.

\section{RESULTS}

My identifications for cortical sulci are indicated on photographs of Einstein's brain (Figure 1). As far as I know, this is the first time that

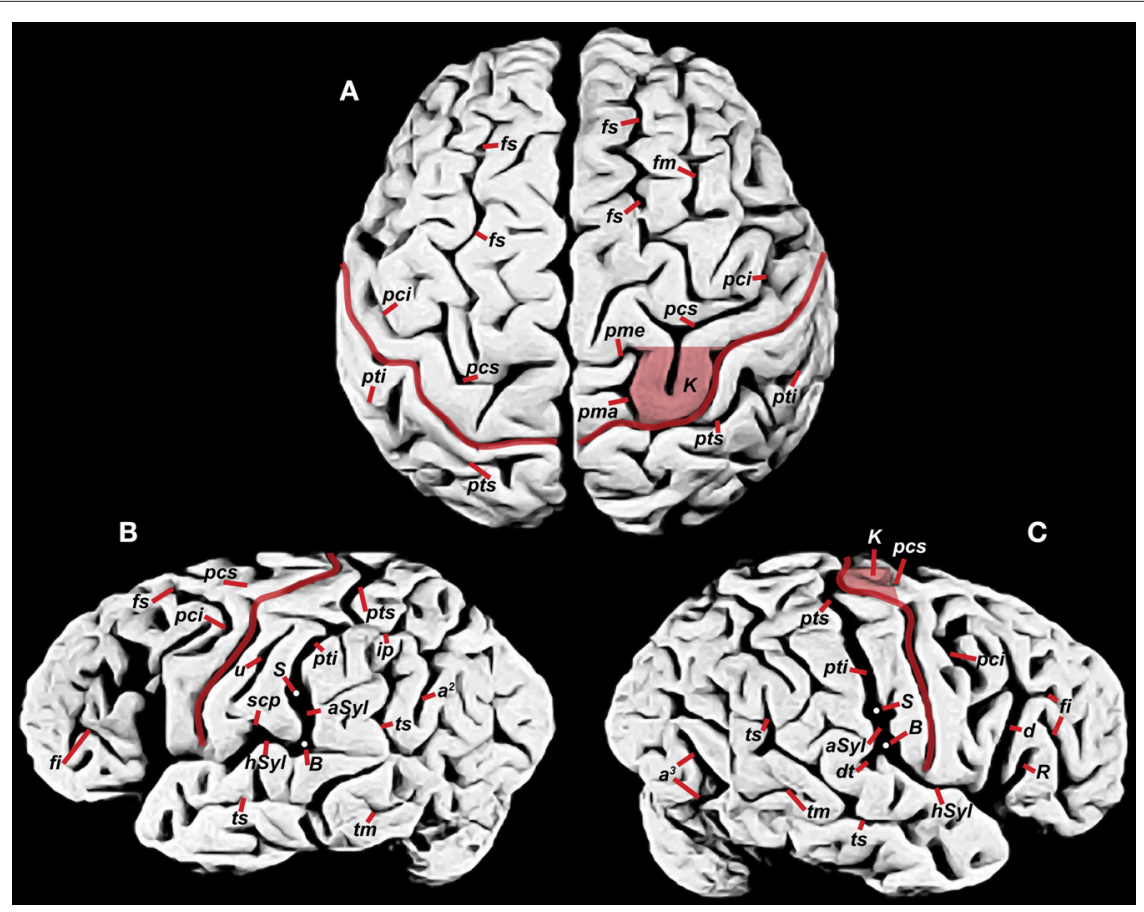

FIGURE 1 | Photographs of Einstein's brain that were taken in 1955, adapted from Witelson et al. (1999b) with identifications added here. (A) Dorsal view, (B) left lateral view, (C) right lateral view. Sulci: angular $\left(a^{2}\right)$, anterior occipital $\left(a^{3}\right)$, ascending limb of the posterior Sylvian fissure (aSy), central fissure (red lines), diagonal $(d)$, descending terminal portion of $a S y l(d t)$, inferior frontal $(f)$, middle frontal $(f m)$, superior frontal $(f s)$, horizontal limb of the posterior Sylvian fissure
( $h$ Sy), intraparietal (ip), precentral inferior and superior (pci, pcs), marginal precentral (pma), medial precentral (pme), postcentral inferior and superior (pti, $p t s)$, ascending ramus of Sylvian fissure (R), subcentral posterior sulcus (scp), middle temporal (tm), superior temporal sulcus (ts), unnamed sulcus in postcentral gyrus ( $u$ ). Other features: branching point between hSyl and aSyl (white dots, B), hand motor cortex knob ( $K$, shaded in $\mathbf{A}, \mathbf{C}$ ), termination of aSyl (white dots, $S$ ). 
the following sulci have been identified on such photographs: angular $\left(a^{2}\right)$, anterior occipital $\left(a^{3}\right)$, diagonal $(d)$, descending terminal portion of the caudal Sylvian $(d t)$, inferior frontal $(f i)$, middle frontal $(f m)$, superior frontal $(f s)$, precentral inferior and superior $(p c i, p c s)$, marginal precentral ( $p m a)$, medial precentral ( $p m e)$, ascending ramus of Sylvian fissure $(R)$, middle temporal $(\mathrm{tm})$, and superior temporal sulcus $(t s)$. The knob $(K)$ representing hand motor cortex (discussed below) is also identified on Einstein's brain for the first time.

The central fissure ( $C$, red in Figure 1$)$ forms the boundary between the postcentral gyrus that represents primary somatosensory cortex and the precentral gyrus that contains motor cortex. On the lateral surface of the brain, Einstein's postcentral gyri were noticeably wider at their lateral compared to medial ends (Figures 1B,C), contrary to measurements from 25 human cadavers, which revealed that the widths of pre- and postcentral gyri are very similar along their entire lengths and manifest little asymmetry between hemispheres (Ono et al., 1990:152-153) (Figure 2). Einstein's left postcentral gyrus contained a long unnamed sulcus $(u)$ parallel to $C$ (Figure 1B), which suggests expansion in depth as well as width in the cortical regions that normally represent face and tongue (Penfield and Rasmussen, 1968). [Although this unnamed sulcus has been identified elsewhere as the retrocentralis transversus ( $r c t$ ) (Witelson et al., 1999a), rct is much shorter and triradiate, as detailed by Connolly (Connolly, 1950:208-209).]

The medial parts of Einstein's sensory/motor strip manifested several unusual features (Figure 1A): On both sides, the precentral superior and inferior sulci ( $p c s$ and $p c i$ ) were continuous, contrary to the normal condition in which the precentral sulcus is separated into two or more segments that characterized $98 \%$ of the 50 hemispheres scored by Ono et al. (1990:43). The medial extent of the left postcentral gyrus was unusually narrow (compare Figures 1 and 2), while on the right it was interrupted by a knob-shaped fold of precentral gyrus, or 'knob' ( $K$; shaded in Figures $1 \mathrm{~A}, \mathrm{C}$ ), that protruded into $C$, causing the latter's middle 'knee' (genu) to merge superficially with the postcentral superior sulcus ( $p t s)$. Although this knob of hand motor cortex is usually better defined in deeper planes (Caulo et al., 2007; Yousry et al., 1997), it sometimes appears on the brain's surface in the perirolandic region as an 'omega sign' (Bangert and Schlaug, 2006), which was the case for Einstein's right hemisphere ( $K$; Figure 1A). Functional imaging studies on normal humans reveal that the knob extends from the surface to the base of the precentral gyrus, and is typically larger in the hemisphere that is contralateral to the preferred hand (Caulo et al., 2007; Volkmann et al., 1998). Although Einstein was right-handed (Wolff and Goodman, 2007), his superficial knob appeared larger in the right hemisphere, which may have been related to his musical training (see below).

My identifications of postcentral superior ( $p t s)$ and inferior (pti) are based on their relationship to the intraparietal sulcus (ip) (Connolly, 1950). As reported by Witelson et al. (1999b), Einstein's $p t i$, which defines the caudal boundary of the postcentral gyrus, connected bilaterally with the termination $(S)$ of the ascending limb of the posterior Sylvian fissure $(a S y l)$, instead of coursing separately and more rostral to $a S y l$ as is typical (Figures $\mathbf{1 B}, \mathbf{C}$ ). Connolly discusses this variation and notes that, although rare, it is more likely to occur on the right hemisphere (Connolly, 1950:210). Additionally, he illustrates this pattern in the left hemisphere of one child (p. 177).

My identification of the level at which aSyl and pti meet in Einstein's right hemisphere [at the white dot labeled $S$ (the caudal termination of the Sylvian fissure) in Figure 1C] is noticeably lower than Witelson et al.'s (1999b, indicated by arrow in Figure 1C). It is not clear when or by whom the arrows were placed on Witelson et al's photographs, or what methods were used to determine the terminal points of the Sylvian fissures. Ideally, one would determine the point $(S)$ of confluence of aSyland ptiby examining their submerged morphology (Connolly, 1950; Steinmetz et al., 1990). Einstein's gross brain is no longer available, however, and there is no indication that the relevant blocks/sections were analyzed for submerged sulcal patterns. My location for right $S$ is based on a number of observations: In lateral view, the sulcus at this level appears relatively wide compared to the medially located and slightly arched $p t i$ that merges with it, similar to Einstein's left hemisphere (Figures 1B,C). These locations result in lengths and positions of $p t s$ and $p t i$ that are relatively balanced in the two hemispheres (Figure 1), and yield an aSyl

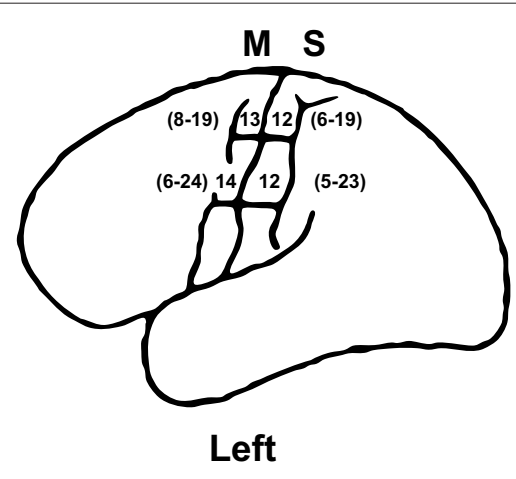

FIGURE 2 |Widths of pre- and postcentral gyri ( $\mathrm{mm}$ ) in left and right hemispheres from $\mathbf{2 5}$ human cadavers. Mean widths and ranges are summarized from Ono et al. (1990, pp. 152-153). S, primary somatosensory cortex; $M$, motor cortex. As illustrated, the range for the medial measurement for the right $M$ is 9-22 $\mathrm{mm}$ rather than 9-12 mm, which is a typo in Ono et al.

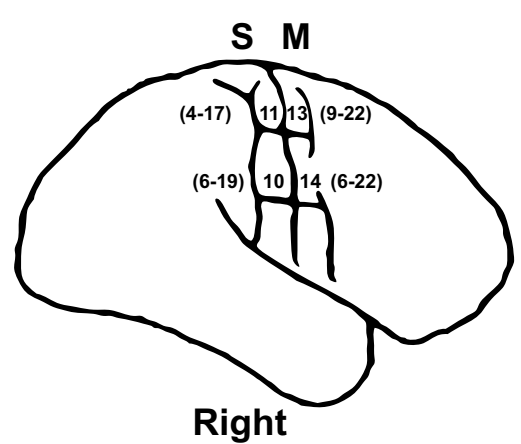

(C. D. Abernathey, personal communication). Note that, contrary to Einstein's brain in which the postcentral gyri are noticeably wider in their lateral compared to medial ends, particularly in the left hemisphere (Figures $1 B, C$ ), the widths of pre- and postcentral gyri in normal individuals are very similar along their entire lengths and manifest little asymmetry between hemispheres. 
that is shorter in the right than left hemisphere, consistent with a statistically significant finding in a 3D MR study on eight normal volunteers (Falk et al., 1991). A descending terminal ( $d t$ ) portion of aSyl appears in Einstein's right hemisphere (compare with Ono et al., 1990:148, photograph C).

The distance between the lateral ends of $C$ and $p t i$ (which coincides with $S$ in Einstein's brain) appears shorter on the right than left side, which is consistent with the reported distance of $3.5 \mathrm{~cm}$ between these two points in Einstein's left hemisphere and $2.0 \mathrm{~cm}$ in the right hemisphere [measurement 22 in Witelson et al.'s (1999b) Table]. On the other hand, Witelson et al's lateral photographs of Einstein's brain (Witelson et al., 1999b:Figures 1B,C) reveal an obviously longer distance between the lateral ends of $C$ and $p t i(=S$ indicated by arrow) on the right, which contradicts measurement 22 in their Table. The lateral end of Einstein's left $C$ is also more rostral than its counterpart on the right (compare Figures 1B,C, this paper), which is another significant asymmetry that characterizes normal people (Falk et al., 1991). These data suggest that the correct location for $S$ in Einstein's right hemisphere is the one presented here.

Of 58 brains from normal humans that could be scored bilaterally, a pattern of pti connecting with $S$ similar to Einstein's appeared in $8(13.7 \%)$ of the right hemispheres and $1(1.7 \%)$ of the left hemispheres, but never appeared in both hemispheres of the same brain (Steinmetz et al., 1990). If one generalizes from this sample and makes the assumption that the left and right occurrences are independent, then the odds of it occurring on both sides of one individual's brain (as it did on Einstein's) are $0.137 \times 0.017=0.002$ (or 0.2 of $1 \%$ ). Because of this unusual sulcal pattern, Einstein's aSyls were not capped by continuous supramarginal gyri (Figure 3) that represent an important language area in the left hemisphere, Brodmann's area 40 (BA 40, Brodmann, 1909). As discussed below, this morphology may have been related to Einstein's delayed acquisition and use of language.

\section{DID EINSTEIN HAVE PARIETAL OPERCULA?}

Whether or not Einstein had parietal opercula has been debated (Galaburda, 1999; Witelson et al., 1999a,b). The term 'operculum' derives from the Latin operire, which means to close or shut and refers to a lid or cover. Cortical opercula begin to develop around the fifth month in the human fetus as parietal, temporal and, eventually, frontal cortices expand and gradually cover the (previously) exposed insula (Connolly, 1950). Normally, the parietal operculum is located between the lateral end of $C$ and the termination of $a S y l$ and includes lateral portions of the inferior postcentral gyrus (BA 43) and rostral supramarginal gyrus (BA 40) (Steinmetz et al., 1990) (Figure 3A), although most of it is buried on the superior bank within the Sylvian fissure (Eickhoff et al., 2006).

It is reasonable to speculate that the supramarginal gyrus began to develop prenatally in Einstein's left hemisphere but was subsequently divided when pti merged with $a S y l$, consistent with Connolly's discussion of the order in which prenatal opercula and postcentral sulci develop (Connolly, 1950). Numerous landmarks suggest that the cortex directly rostral to $a S y l$ in Einstein's brain was part of BA 40 and that a separate portion of this area also occurred caudal to $a S y l$ above an unnamed sulcus stemming from the branching point $(B)$ in the left hemisphere at the junction of the horizontal limb of the posterior Sylvian fissure $(h S y l)$ and $\operatorname{aSyl}$ (Brodmann, 1909; Eickhoff et al., 2006; Steinmetz et al., 1990) (Figure 3B). BA 40 and 43 normally share a border near the subcentral posterior sulcus ( $s c p$ ) (Brodmann, 1909; Eickhoff et al., 2006), which is present on Einstein's left hemisphere and permits tentative identification of BA 43 in addition to BA 40 (Figure 3B). These observations support Galaburda's identification of a left parietal operculum in Einstein's brain (Galaburda, 1999).

Einstein's right insula is also covered with an operculum that probably contained BA 43 which may, or may not, have extended onto the brain's lateral surface (Eickhoff et al., 2006) (Figure 1C).

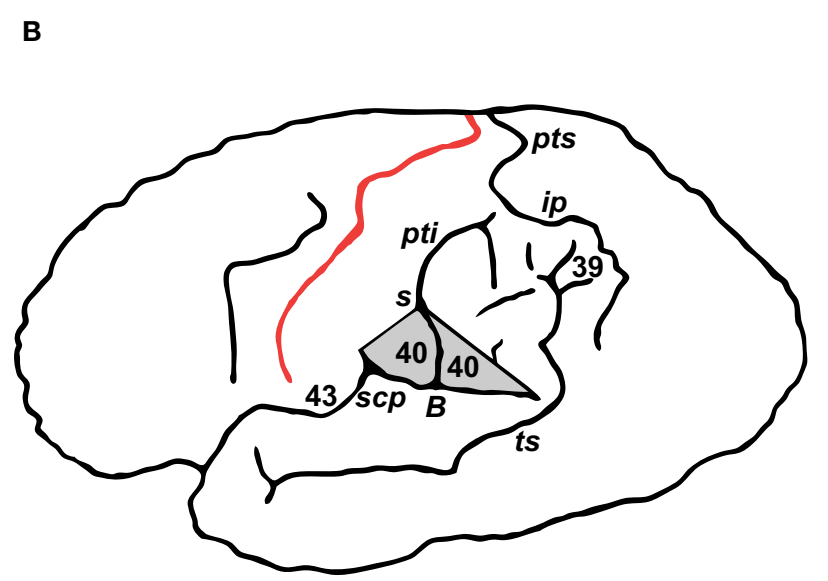

caudally from B. (B) These landmarks are available in Einstein's left hemisphere and enclose an area that probably approximates a minimal surface representation of BA 40. A supramarginal gyrus containing BA 40 did not cap aSyl, however, because the latter was continuous with pti. 
One would need to examine the cytoarchitecture within the superior bank of the Sylvian fissure in this hemisphere to determine whether the operculum also contained part of BA 40 (Eickhoff et al., 2006). Given how the brain was processed, however, it seems unlikely that such a study could be done.

\section{DISCUSSION}

Exceptional abilities are sometimes manifested in cortical symmetries or asymmetries that depart from those of normal controls (Amunts et al., 2004). For example, the better performances of musicians with perfect pitch are associated with relatively enlarged left planum temporale (Schlaug et al., 1995), and early commencement of musical training is sometimes associated with pronounced structural differences in hand representations (Schlaug, 2001) that are reflected in cortical sulcal patterns (Bangert and Schlaug, 2006). Professional keyboard players have deeper and more symmetrical (in terms of depth) central sulci within their sensorimotor hand representations than normal controls, which appears to be related to increased skill of the nondominant hand as a result of early training (Amunts et al., 1997; Jancke et al., 1997). The cytoarchitecture of BA 44 was more symmetric in the polyglot Emil Krebs than in controls, while that of his BA 45 was more asymmetric than theirs (Amunts et al., 2004).

Einstein's brain was characterized by an unusual mixture of symmetrical and asymmetrical features. A rare convergence of the postcentral sulcus with the Sylvian fissure (Steinmetz et al., 1990) occurred bilaterally in Einstein's brain (Witelson et al., 1999b), which nonetheless manifested a marked degree of asymmetry in the width of the lateral postcentral gyrus that favored the left hemisphere, and a pronounced knob in the right hemisphere. These asymmetries together with an atypical lack of uniformity in the medial and lateral widths of the pre-and post central sulci (Ono et al., 1990) indicate that the gross anatomy of Albert Einstein's brain in and around the primary somatosensory and motor cortices was, indeed, unusual.

Musicians have more pronounced cortical knobs than nonmusicians, and right-handed string-players (as opposed to pianists) tend to have differentially pronounced superficial knobs on their right rather than left hemispheres, especially if they began their musical training early in life (Bangert and Schlaug, 2006). Correspondingly, somatosensory representations for the left digits of right-handed violinists are larger than those of controls (especially if they began training before the age of 12), presumably because their performances engage their left digits more than the right ones that manipulate the bow (Elbert et al., 1995). Einstein's differentially enlarged knob $(K)$ on the surface of his right hemisphere is consistent with the fact that he was a right-handed stringplayer who took violin lessons from age 6 to 14 years (Bangert and Schlaug, 2006; Bucky, 1992). Although there is no proof that musical training induces changes in hand representations of musicians, the correlation between early commencement of training and pronounced structural differences (Amunts et al., 1997), including superficially prominent knobs (Bangert and Schlaug, 2006), suggests that these features developed in response to individuals' lifetime experiences (Schlaug, 2001), which does not rule out a genetic component.
An earlier report concluded that Einstein's visuospatial and mathematical cognition may have been influenced by relatively expanded parietal regions (Witelson et al., 1999b). Recent neuroanatomical and functional imaging studies suggest, further, that cortical features reported here may also have been related to Einstein's self-reported preference for thinking in sensory impressions including visual images rather than words (Einstein, 1970). Normally, BA 40 is one continuous region that is involved with short-term maintenance of phonemes and syllables during language tasks (Galaburda et al., 2002), and damage to the left supramarginal gyrus and its underlying white matter may result in a profound disability in which 'words are no longer a means of expression of thoughts, although the individual may still be capable of thinking' (Crosby et al., 1962). It is therefore tempting to speculate that the unusual superficial cleaving of BA 40 and seamless melding of its rostral portion with the postcentral gyrus in Einstein's left hemisphere (Figures 1B and 3B) may have been associated with his well-known delay at acquiring language and the fact that he repeated sentences to himself softly until the age of about seven (Wolff and Goodman, 2007).

As an adult, Einstein famously observed that 'the words or the language, as they are written or spoken, do not seem to play any role in my mechanism of thought. The psychical entities which seem to serve as elements in thought are certain signs and more or less clear images which can be 'voluntarily' reproduced and combined' (Hadamard, 1945). Einstein laughed when informed that many people always think in words (Wertheimer, 1959), and emphasized that concepts became meaningful for him 'only through their connection with sense-experiences' (Einstein, 1970). He was a synthetic thinker. Family members and friends have documented that, when stuck on a physics problem, Einstein would play the violin until, suddenly, he would announce excitedly, 'I've got it!' (Bucky, 1992). It is interesting to contemplate that such synthesizing may have contributed to Einstein's insights, and that his extraordinary abilities may, to some degree, have been associated with the unusual gross anatomy of his cerebral cortex in and around the primary somatosensory and motor cortices. Although these views are speculative, the identifications of previously unrecognized cortical morphology on Einstein's brain will, hopefully, be of use to future scholars who have access to new information and methodologies.

\section{ACKNOWLEDGEMENTS}

Marc Bangert of Harvard Medical School provided feedback on an earlier version of this paper. For other helpful correspondence, I thank neurosurgeon C. D. Abernathey; Albert Galaburda of the Beth Deaconess Medical Center, Boston; Elliot Krauss of University Medical Center, Princeton; Frederick E. Lepore of Robert Wood Johnson Medical School, New Jersey; and Barbara Wolff of the Albert Einstein Archives, Jewish National \& University Library, Jerusalem. Kathryn O'Donnell helped with manuscript preparation, and Jonathan Lewis assisted in preparation of the images. This paper was completed while I was a 2008-2009 visiting scholar at the School for Advanced Research (SAR) in Santa Fe, New Mexico, for which I am deeply appreciative to SAR and its president, James Brooks. 


\section{REFERENCES}

Amunts, K., Schlaug, G., Jancke, L., Steinmetz, H., Schleicher, A., Dabringhaus, A., and Zilles, K. (1997). Motor cortex and hand motor skills: structural compliance in the human brain. Hum. Brain Mapp. 5 , 206-215.

Amunts, K., Schleicher, A., Burgel, U., Mohlberg, H., Uylings, H. B., and Zilles, K. (1999). Broca’s region revisited: cytoarchitecture and intersubject variability. J. Comp. Neurol. 412, 319-341.

Amunts, K., Schleicher, A., and Zilles, K. (2004). Outstanding language competence and cytoarchitecture in Broca's speech region. Brain Lang. 89, 346-353.

Anderson, B., and Harvey, T. (1996). Alterations in cortical thickness and neuronal density in the frontal cortex of Albert Einstein. Neurosci. Lett. 210, 161-164.

Bangert, M., and Schlaug, G. (2006). Specialization of the specialized in features of external human brain morphology. Eur. J. Neurosci. 24, 1832-1834.

Brodmann, K. (1909). Vergleichende Lokalisationslehre der Grosshirnindein ihren Prinzipien Dargestellt auf Grund des Zellenbaues. Leipzig, Barth.

Bucky, P. A. (1992). The Private Albert Einstein. Kansas City, Andrews and McMeel.

Caulo, M., Briganti, C., Mattei, P. A., Perfetti, B., Ferretti, A., Romani, G. L., Tartaro, A., and Colosimo, C. (2007). New morphologic variants of the hand motor cortex as seen with MR imaging in a large study population. AJNR Am. J. Neuroradiol. 28, 1480-1485.

Connolly, J. C. (1950). External Morphology of the Primate Brain. Springfield, IL, C. C. Thomas.

Crosby, E., Humphrey, T., and Lauer, E. W. (1962). Correlative Anatomy of the Nervous System. New York, Macmillan.
Diamond, M. C., Scheibel, A. B., Murphy, G. M. Jr., and Harvey, T. (1985). On the brain of a scientist: Albert Einstein. Exp. Neurol. 88, 198-204.

Eickhoff, S. B., Schleicher, A., Zilles, K., and Amunts, K. (2006). The human parietal operculum. I. Cytoarchitectonic mapping of subdivisions. Cereb. Cortex 16, 254-267.

Einstein, A. (1970). Autobiographical notes. In:Albert Einstein: PhilosopherScientist, Vol. II, The Library of Living Philosophers, 3rd Edn, P. A. Schilpp, eds (La Salle, IL, Open Court Press).

Elbert, T., Pantev, C., Wienbruch, C., Rockstroh, B., and Taub, E. (1995). Increased cortical representation of the fingers of the left hand in string players. Science 270, 305-307.

Falk, D. (1982). Mapping fossil endocasts. In: Primate Brain Evolution: Methods and Concepts, E. Armstrong and D. Falk, eds (New York, Plenum), pp. 217-226.

Falk, D., Hildebolt, C., Cheverud, J., Kohn, L. A., Figiel, G., and Vannier, M. (1991). Human cortical asymmetries determined with 3D MR technology. J. Neurosci. Methods 39, 185-191.

Galaburda,A.M. (1999). Albert Einstein's brain. Lancet 354, 1821; author reply 1822.

Galaburda, A. M., Kosslyn, S. M., and Christen, Y. (2002). The Languages of the Brain. Cambridge, MA, Harvard University Press.

Gould, S. J. (1981). The Mismeasure of Man. New York, WW Norton.

Hadamard, J. (1945). The Psychology of Invention in the Mathematical Field. Princeton, Dover Publications.

Hines, T. (1998). Further on Einstein's brain. Exp. Neurol. 150, 343-344.

Jancke, L., Schlaug, G., and Steinmetz, H. (1997). Hand skill asymmetry in professional musicians. Brain Cogn. $34,424-432$.

Jerison, H. J. (1973). Evolution of the Brain and Intelligence. New York, Academic Press.
Lepore, F. E. (2001). Dissecting Genius: Einstein's Brain and the Search for the Neural Basis of Intellect, Vol. 3. New York, Dana Press, pp. 11-26.

Magnotta, V. A., Andreasen, N. C., Schultz, S. K., Harris, G., Cizadlo, T., Heckel, D., Nopoulos, P., and Flaum, M. (1999). Quantitative in vivo measurement of gyrification in the human brain: changes associated with aging. Cereb. Cortex 9, 151-160.

Ono, M., Kubik, S., and Abernathy, C. D. (1990).Atlas of the Cerebral Sulci. New York, Hafner Publishing Company.

Penfield, W., and Rasmussen, T. (1968). The Cerebral Cortex of Man: A Clinical Study of Localization of Function. New York, Hafner Publishing Company.

Roth, G., and Dicke, U. (2005). Evolution of the brain and intelligence. Trends Cogn. Sci. 9, 250-257.

Schlaug, G. (2001). The brain of musicians. A model for functional and structural adaptation. Ann. NY Acad. Sci. 930, 281-299.

Schlaug, G., Jancke, L., Huang, Y., and Steinmetz, H. (1995). In vivo evidence of structural brain asymmetry in musicians. Science 267, 699-701.

Shaw, P., Greenstein, D., Lerch, J. Clasen, L., Lenroot, R., Gogtay, N., Evans, A., Rapoport, J., and Giedd, J. (2006). Intellectual ability and cortical development in children and adolescents. Nature 440, 676-679.

Steinmetz, H., Ebeling, U., Huang, Y. X., and Kahn, T. (1990). Sulcus topography of the parietal opercular region: an anatomic and MR study. Brain Lang. $38,515-533$.

Volkmann, J., Schnitzler, A., Witte, O. W. and Freund, H. (1998). Handedness and asymmetry of hand representation in human motor cortex. $J$. Neurophysiol. 79, 2149-2154.

Welker, W. I., and Campos, G. B. (1963). Physiological significance of sulci in somatic sensory cerebral cortex in mammals of the family procyonidae. J. Comp. Neurol. 120, 19-36.
Wertheimer, M. (1959). Productive Thinking. New York, Harper.

Witelson, S. F., Kigar, D. L., and Harvey T (1999a). Authors' reply. Lancet 354, 1822.

Witelson, S. F., Kigar, D. L., and Harvey T (1999b).The exceptionalbrain of Albert Einstein. Lancet 353, 2149-2153.

Wolff, B., and Goodman, H. (2007). The legend of the dull-witted child who grew up to be a genius. Available at: http://www.albert-einstein.org/ article_handicap.html

Yousry, T. A., Schmid, U. D., Alkadhi, H., Schmidt, D., Peraud, A., Buettner, A., and Winkler, P. (1997). Localization of the motor hand area to a knob on the precentral gyrus. A new landmark. Brain 120(Pt 1), 141-157.

Zilles, K., Schleicher, A., Langemann, C. Amunts, K., Morosan, P., PalomeroGallagher, N., Schormann, T. Mohlberg,H.,Burgel,U.,Steinmetz, H., Schlaug, G., and Roland, P. E. (1997). Quantitative analysis of sulci in the human cerebral cortex: development, regional heterogeneity, gender difference, asymmetry, intersubject variability and cortical architecture. Hum. Brain Mapp. 5, 218-221.

Conflict of Interest Statement: The author declares that the research was conducted in the absence of commercial or financial relationships that could be construed as a potential conflict of interest.

Received: 27 February 2009; paper pending published: 02 April 2009; accepted: 07 April 2009; published online: 04 May 2009. Citation: Falk D (2009) New information about Albert Einstein's brain. Front. Evol. Neurosci. (2009) 1:3. doi: 10.3389/neuro.18.003.2009

Copyright (๑) 2009 Falk. This is an openaccess article subject to an exclusive license agreement between the authors and the Frontiers Research Foundation, which permits unrestricted use, distribution, and reproduction in any medium, provided the original authors and source are credited. 